\title{
"GENERAZIONI" DI DIRITTI UMANI E DIRITTI UMANI DELLE GENERAZIONI FUTURE
}

\author{
Nota del s.c. FAUSTO POCAR (*)
}

(Adunanza del 1 marzo 2018)

SUNTO. - La classificazione dei diritti umani in "generazioni", ormai abituale nella letteratura giuridica, appare inadeguata dato che si tratta di diritti inerenti alla persona che non trovano la loro esistenza nella loro disciplina giuridica che viene in considerazione solo ai fini della loro protezione, come si ricava chiaramente dalla Dichiarazione universale del 1948. Questa, inoltre, comprende i diritti diffusi della c.d. terza generazione (diritto alla pace, allo sviluppo, all'ambiente) in una disposizione relativa a un ordine sociale e internazionale che porta a considerarli più che diritti, espressione di un dovere inteso a permettere l'esercizio dei diritti umani. Una lettura diacronica del carattere universale della Dichiarazione implica infine che tale dovere si estenda alla garanzia dei diritti non solo della presente, ma anche delle future generazioni di esseri umani.

$$
* * *
$$

ABSTRACT. - The classification of human rights in "generations", habitual in legal litterature, looks inappropriate in light of the consideration that human rights are inherent in human beings and do not depend on their recognition under the law, which is only relevant for their protection, as it clearly derives from the 1948 Universal Declaration. Furthermore, the Declaration refers to diffused rights of the so called third generation (right to peace, to development, to environment) under a provision on a social and international order, which leads to deny their nature as rights and to conclude that they should rather be qualified as duties aimed at ensuring the exercise of human rights. Finally, a diachronic reading of the universal nature of the Declaration implies that such duties comprise an international guarantee of human rights not only of the present, but also of future generations of human beings.

È ormai abituale, nella letteratura giuridica degli ultimi decenni sui diritti fondamentali della persona, di classificarli in termini di generazioni, suddividendoli secondo le loro caratteristiche e attribuendoli

(*) Università degli Studi di Milano, Italy. E-mail: fausto.pocar@unimi.it 
all'una o all'altra generazione. In particolare, i diritti civili e politici sarebbero in quest'ottica diritti della prima generazione, quelli economici, sociali e culturali apparterrebbero alla seconda generazione, mentre alla terza generazione apparterrebbero i diritti diffusi più recentemente definiti nel diritto internazionale, quali il diritto alla pace, allo sviluppo, a un ambiente sicuro e salubre, o all'uso delle risorse naturali. Sarebbe inoltre in via di formazione una quarta generazione di diritti, concernente in particolare la c.d. società dell'informazione.

Questa classificazione appare formalmente basata sugli strumenti giuridici internazionali esistenti adottati nel quadro delle Nazioni Unite a partire dalla Dichiarazione universale dei diritti dell'uomo del 10 dicembre 1948. Da una parte sono abitualmente indicati come diritti della prima e seconda generazione quelli previsti nei due Patti del 16 dicembre 1966, rispettivamente dedicati ai diritti civili e politici e ai diritti economici, sociali e culturali. Dall'altra, i diritti della terza generazione sono quelli considerati da vari strumenti specifici, per lo più dichiarazioni dell'Assemblea generale delle Nazioni Unite, dato che il loro riconoscimento è recente e le incertezze che hanno accompagnato la loro definizione hanno finora impedito l'adozione di uno strumento che tratti della loro protezione in modo complessivo.

Come tutte le classificazioni, quella ora richiamata non si presta ad una rigida separazione delle categorie di diritti individuati. Se, come si può ricavare dai Patti, la distinzione fra la prima e la seconda generazione sta nel fatto che i diritti della prima hanno efficacia diretta e comportano un obbligo immediato degli Stati di rispettarli, mentre quelli della seconda richiedono un'attuazione progressiva e creano quindi un obbligo non immediato di rispettarli nella loro interezza, non può dimenticarsi che non tutti i diritti si riconoscono perfettamente in questa descrizione. Altrettanto è a dirsi per i diritti della terza generazione, talora comprensivi, come il diritto allo sviluppo, di elementi propri di diritti delle altre categorie. Ma, nonostante questa possibile critica, tutto sommato di scarso rilievo, della classificazione in esame, questa ha certamente il merito di indicare che la suddivisione dei diritti in generazioni riflette il progressivo riconoscimento di gruppi di diritti e la necessità di misure diverse di attuazione. Tuttavia, resta piuttosto da dimostrare che una classificazione di diritti in termini di "generazioni" sia auspicabile per una corretta comprensione della natura e dell'essenza dei diritti fondamentli della persona umana. Mi permetto di sostenere che una classificazione di questo genere appare, nella materia qui considerata, inappropriata e fuorviante. 
Va in primo luogo osservato che il termine "generazione" è palesemente inidoneo a descrivere categorie di diritti fondamentali. Esso ha infatti una connotazione temporale, che implica una successione di situazioni, in base alla quale quando una nuova generazione viene ad esistenza, la precedente viene superata. In questo schema, la generazione più vecchia viene progressivamente messa da parte per fare spazio alla più giovane, fino ad essere sostituita da quest'ultima. È tuttavia, o almeno dovrebbe essere, evidente che in materia di diritti fondamentali della persona, quando emerge una nuova cosiddetta generazione, i nuovi diritti che in questa si riconoscono vengono ad aggiungersi a quelli riconosciuti e protetti come appartenenti a una generazione precedente. A meno che non si voglia sostenere che con l'emergere di una nuova generazione i diritti precedenti vengono ad essere ricompresi in quelli nuovi e vi perdono la loro identità. Se questa posizione è stata talora fatta valere, a favore della protezione di diritti collettivi piuttosto che di diritti individuali, essa non può certo dirsi prevalente nella pratica internazionale, che continua a preoccuparsi della protezione anche dei diritti delle generazioni precedenti in quanto tali. La preoccupazione maggiore che solleva una impostazione in termini di "generazioni" non è soltanto terminologica. È piuttosto il timore di abusi che possano condurre, e hanno talora condotto, ad affermare posizioni secondo le quali nuovi diritti, soprattutto diritti collettivi, vengono considerati esclusivi di diritti di generazioni precedenti, o quanto meno acquistano un carattere prioritario rispetto ad essi.

In secondo luogo, anche se una impostazione generazionale può apparire a prima vista corretta per una classificazione in una prospettiva storica, uno studio più approfondito mostra che essa è erronea anche dal punto di vista storico. L'errore è dovuto a una lettura non corretta della Dichiarazione universale dei diritti dell'uomo. Questa, come è affermato dai più autorevoli giuristi, non può essere considerata un elenco di diritti presentati in modo non organizzato, divisi in gruppi diversi che vengono ad esistenza quando la loro protezione si sviluppa progressivamente nel contesto nazionale ed internazionale. Al contrario, la Dichiarazione universale deve essere intesa come un documento coerente nel quale tutti i diritti elencati sono indivisibili, interconnessi e interdipendenti. Come si esprime il primo considerando del preambolo, la Dichiarazione rappresenta una espressione e una specificazione giuridica del riconoscimento della dignità inerente all'essere umano, come fondamento della libertà, della giustizia e della pace nel mondo. 
In questo contesto, i diritti delle cosiddette prima e seconda generazione sono indicati espressamente nel documento, mentre i diritti della cosiddetta terza generazione, pur non espressamente indicati, possono in larga misura, e forse completamente, trovare la loro espressione nella disposizione generale dell'art. 28, secondo il quale "ciascuno ha diritto a un ordine sociale e internazionale nel quale i diritti e le libertà affermati in questa Dichiarazione possano essere pienamente realizzati".

Inoltre, un riferimento a "generazioni" trascura il fatto che i diritti fondamentali della persona non trovano, nella Dichiarazione universale, il loro fondamento nel diritto positivo, ma sono piuttosto inerenti agli esseri umani e, come tali, devono essere riconosciuti e protetti dal diritto. Il primo considerando della Dichiarazione intenzionalmente si riferisce al "riconoscimento... dei diritti uguali e inalienabili di tutti i membri della famiglia umana", sul presupposto che questi diritti non derivano la loro esistenza dal diritto positivo, ma preesistono al loro riconoscimento e alla loro protezione da parte del diritto vigente nell'ordinamento interno ed internazionale. C'è un'evidente contraddizione nel riferimento a "generazioni" di diritti e nella contemporanea affermazione che tali diritti sono inerenti agli esseri umani come tali, vale a dire che ciascuno ne è titolare per il semplice fatto della nascita. Un riferimento a generazioni significherebbe infatti che i diritti fondamentali possono cambiare secondo la generazione a cui appartengono, e che dunque anche il fondamento della loro titolarità può mutare. Un simile riferimento confonderebbe pertanto l'essenza della titolarità dei diritti della persona con la loro protezione secondo la legge. Mentre la prima non cambia, la seconda può essere ed è soggetta a mutamenti, dato che l'ampiezza del riconoscimento e della protezione loro accordata dipende dal diritto applicable in un determinato ambito spaziale e temporale.

Alla luce delle considerazioni che precedono, sembra doveroso abbandonare una classificazione in termini di "generazioni" di diritti e fare piuttosto riferimento a fasi diverse e successive del progressivo riconoscimento giuridico formale dei diritti della persona e della protezione loro accordata secondo il diritto interno ed internazionale.

Se ora si pone mente alla relazione esistente tra i gruppi di diritti sopra menzionati, l'interdipendenza fra i diritti civili e politici da un lato e i diritti economici, sociali e culturali dall'altro è stata ampiamente messa in luce dalla dottrina giuridica ed è chiaramente espressa dalla Dichiarazione universale e da numerose risoluzioni dell'Assemblea 
generale delle Nazioni Unite. Questi documenti mostrano anche che la distinzione fra i due gruppi di diritti non è basata su un riferimento ad una sequenza temporale quanto al loro riconoscimento e alla loro protezione. Più delicata è invece la definizione della relazione esistente tra questi due gruppi di diritti presi congiuntamente e la terza categoria di diritti diffusi, impropriamente chiamati della terza generazione.

Va osservato a questo proposito che questi diritti, a causa del loro carattere di diritti diffusi, presentano una dimensione collettiva che appare prevalente rispetto a quella individuale. In tema di diritti fondamentali, vi è tuttavia sempre un pericolo nel riconoscimento di diritti collettivi, quello di diluirne il contenuto facendoli dipendere dall'interesse superiore della società. Se è naturale che i diritti individuali devono essere in armonia con l'interesse collettivo, essi non devono avere un ruolo subordinato a tale interesse che ne annulli il contenuto e la loro natura di essere inerenti alla persona che ne è titolare. In altre parole, non deve mai essere superata la soglia al di là della quale essi risultino interamente sacrificati sull'altare dell'interesse collettivo; quest'ultimo dovrebbe trovare protezione solo nella misura in cui sia il risultato di una considerazione dei diritti individuali di tutti i membri del gruppo.

Per evitare il rischio di diluire i diritti individuali oltre un certo punto, c'è da chiedersi se non sia necessaria una certa prudenza prima di qualificare questi diritti diffusi alla stregua di diritti fondamentali paragonabili a quelli contenuti nella Dichiarazione universale e nei Patti, o negli altri strumenti internazionali che li riguardano. Infatti questi diritti potrebbero meglio essere considerati mezzi per assicurare il rispetto e il godimento dei diritti individuali, piuttosto che diritti fondamentali essi stessi. La pace, un ambiente sicuro e sano, lo sviluppo, e un'equa distribuzione delle risorse sono certamente condizioni necessarie per il godimento dei diritti fondamentali e delle libertà individuali, ma è dubbio che sia corretto definirli come diritti umani ulteriori a sé stanti, inerenti alla natura umana. Una impostazione di questo genere implica tuttavia che si affermi il dovere della società, e in particolare ma non solo della comunità internazionale, di garantire l'esistenza di queste condizioni, in modo che i diritti individuali fondamentali possano essere goduti ed esercitati da coloro che ne sono titolari.

Si tratta di un'impostazione che non è incompatibile con meccanismi e procedure con le quali agli individui sia attribuita la capacità di chiedere e ottenere misure per assicurare il mantenimento della pace, la conservazione dell'ambiente, l'adozione di politiche di sviluppo, o 
un'equa distribuzione delle risorse. A questo fine non sembra indispensabile definire queste situazioni come oggetto di diritti fondamentali, dato che la loro relazione con diritti individuali è sufficiente per giustificare meccanismi e procedure di questo genere.

La qualificazione dei cosiddetti diritti della terza generazione come condizioni per garantire il godimento dei diritti individuali piuttosto che come ulteriori diritti, unitamente all'accento posto sull'obbligo di attuare tali condizioni, solleva il problema della definizione del contenuto di tale obbligo. Quest'ultimo è chiaramente collegato all'attuazione della Dichiarazione universale e al significato da attribuire alla parola "universale". Questo termine è generalmente inteso come riferito al riconoscimento dei diritti fondamentali come inerenti a tutti gli esseri umani senza distinzione di alcun genere, e senza considerazione del paese o territorio in cui una persona si trova. Lo stesso art. 2 della Dichiarazione universale conferma questo significato, e non permette di dubitare che la parola "universale" implichi che ogni essere umano è titolare dei diritti in essa proclamati.

Vi è tuttavia da chiedersi se all'universalità debba essere attribuita una dimensione puramente orizzontale, che comporti l'applicazione della Dichiarazione a ciascuno in ogni luogo in un determinato momento storico, o se invece essa non abbia anche una dimensione verticale, che implica il riconoscimento dei diritti di generazioni future, e pertanto anche l'obbligo di conservare le condizioni necessarie per il loro godimento.

A me sembra che debba essere affermato questo significato bidimensionale, nel senso che anche la seconda dimensione rientra nel concetto di universalità. Se si accetta che i diritti fondamentali sono inerenti alla persona umana, ne consegue che essi non possono appartenere solo ai componenti della società umana attuale, ma devono appartenere anche ai componenti futuri di tale società, e che quindi la Dichiarazione universale deve essere intesa come rivolta a proteggere anche i diritti degli individui appartenenti a generazioni future, anche prima della loro venuta ad esistenza. È ovvio che l'obbligo di assicurare il rispetto e il godimento di tali diritti acquista un rilievo concreto solo al momento della nascita delle persone che ne sono titolari. Ma gli obblighi relativi al mantenimento e alla conservazione delle condizioni essenziali per permettere tanto il godimento quanto l'effettiva attuazione dell'obbligo di garantirli devono avere un significato e una dimensione verticale, o diacronica, tale da applicarsi, in ogni momento storico, non solo con 
riferimento agli individui esistenti in quel tempo, ma anche ai membri futuri della famiglia umana.

Tali obblighi sono pertanto il riflesso di una speciale responsabilità per ogni generazione di individui, una responsabilità che ci riporta all'attuazione di quelli che impropriamente vengono qualificati diritti della terza generazione. Il mancato rispetto degli obblighi che riguardano l'attuazione dei diritti è in contrasto con la Dichiarazione universale e deve essere considerato come una violazione di essa, senza la necessità di creare nuove categorie di diritti. L'accento deve essere posto piuttosto sulle responsabilità che comporta l'attuazione dei diritti fondamentali individuali intesa nella sua dimensione universale avente il significato verticale ora indicato.

In questo contesto, non va dimenticato che la comunità internazionale ha sottolineato negli ultimi decenni che, quando ricorrano determinate condizioni, gravi violazioni di diritti umani possono comportare la responsabilita penale individuale delle persone che le hanno commesse, quando in particolare la loro azione od omissione sia intenzionale. Pertanto, la nozione di crimini contro l'umanità non può essere limitata alla sistematica o diffusa violazione dei diritti fondamentali nei confronti degli individui esistenti, ma può anche riferirsi anche a tale violazione quando ne siano colpiti gli appartenenti a generazioni future.

In conclusione, vi sono motivi a favore del punto di vista che non è e non è mai stato opportuno riferirsi ai diritti umani in termini di "generazioni". Ai fini di una progressiva protezione dei diritti umani appare più appropriato accettare una definizione di universalità con diversi profili, e qualificare i diritti della cosiddetta terza generazione come condizioni per assicurare il godimento dei diritti individuali piuttosto che come una nuova categoria di diritti. La continua proliferazione di categorie di diritti non contribuisce ad aumentare la protezione, ma distoglie l'attenzione dai problemi reali che si presentano in questa materia, costituiti dall'attuazione del bill of rights come espresso nella Dichiarazione universale e nei Patti internazionali sui diritti umani. 
